\title{
Hemolytic Uremic Syndrome: "Diagnosis and Management in Pediatric Population"
}

\author{
Farkhanda Hafeez ${ }^{1}$, Sadaf Asim², Naureen Akhtar ${ }^{3}$, Junaid Ahmad ${ }^{4}$ \\ ${ }^{1}$ Department of Pediatric Nephrology, Bahria International Hospital Orchard, Lahore, Pakistan. \\ ${ }^{2}$ Department of Pediatric Nephrology, National Institute of Child Health, Karachi, Pakistan. \\ ${ }^{3}$ Department of Pediatric Nephrology, The Children's Hospital, Lahore, Pakistan. \\ ${ }^{4}$ Department of Pediatric Nephrology, The Children's Hospital, Faisalabad, Pakistan.
}

\begin{abstract}
:
The review article" Hemolytic Uremic Syndrome: Diagnosis and Management in Pediatric Population” is an update on different types of hemolytic syndrome, its diagnosis and treatment. This write up also sheds light on limitation in its management in a resource limited country and suggestions to overcome it. It is hoped that it will generate interest and will encourage the readers to share their experiences.
\end{abstract}

Key Words: Hemolytic Uremic Syndrome, Thrombotic microangiopathies, Shiga toxin, complement dysregulation, Plasma Exchange, Eculizumab.

\author{
Corresponding Author: \\ Professor Dr. Farkhanda Hafeez \\ Consultant Pediatric Nephrologist \\ Bahria International Hospital Orchard, \\ Lahore, Pakistan. \\ Contact : +920300461930 \\ E.mail : $\underline{\text { drfarkh@hotmail.com }}$
}

Received: October 3, 2020. Accepted October 25, 2020.

PJKD 2020;4(3):338-346

\section{Introduction:}

Hemolytic uremic syndrome (HUS) is one of the thrombotic microangiopathies (TMAs) characterized by nonimmune thrombotic microangiopathic hemolytic anemia, thrombocytopenia and acute kidney injury. ${ }^{1}$ It can be followed by infection with Shiga Toxin producing bacteria, mainly Escherichia Coli (STEC) or may be associated with systemic illness (secondary) or caused mainly by dysregulation of complement system, atypical HUS (aHUS) ( Fig1). ${ }^{2}$ Atypical HUS carries poor prognosis, with first aHUS attack being associated with mortality rate of about $25 \%$ and approximately $50 \%$ of cases resulting in ESRD requiring dialysis.

A variety of clinical conditions have features of TMA and differentiating HUS from other forms of thrombotic microangiopathies is difficult but crucial because of the need for distinct therapeutic approaches. ${ }^{4}$ Fig 2 elaborates how to differentiate atypical hemolytic uremic syndrome (aHUS) from other thrombotic microangiopathies.

HUS remains a diagnostic challenge for nephrologist in developing countries, since direct detection of Shiga toxins by real time PCR, or the detection of antibodies by immunoassay, are not freely available. Likewise, work up of atypical HUS which results from either loss of-function mutations of regulatory genes or gain-of-function mutations in effector genes (C3 and CFB) of alternate pathway (AP) or neutralizing antibodies is not available. ${ }^{6}$

Not only there are diagnostic issues but treatment modalities like facility for plasma exchange and dialysis are available only in couple of centers. Eculizumab, a monoclonal antibody that blocks $\mathrm{C} 5$ activation and terminal membrane attack complex yields good results however, there are financial constraints and availability issues for its usage.

In this article, a brief review of different types of thrombotic microangiopathies will be given with emphasis on diagnostic and treatment modalities of aHUS and suggestions in a resource limited country like Pakistan.

\section{Definition:}

According to "Consensus guidelines for hemolytic uremic syndrome in a developing country" by Bagga. A et al, probable diagnosis of HUS is made if: triad of microangiopathic non immune hemolytic anemia(Hb<10gm/dl) with high $\mathrm{LDH}>450 \mathrm{iu} / \mathrm{L})$, low haptoglobin $(<30 \mathrm{mg} / \mathrm{dl})$, reticulocytosis $(>2 \%)$, presence of schistocytes (upto $10 \%)$ on peripheral smear, thrombocytopenia (Plt $<150,000 / \mu \mathrm{L}$ and acute kidney injury( S/Creatinine 1.5 times higher than reference value for age and gender). 


\section{Types of Hemolytic Uremic Syndrome: \\ Infection-induced HUS:}

Infection-induced HUS is a common cause of acute kidney injury in children and leads to significant morbidity and mortality during the acute phase of the disease. In addition, long-term renal and extra renal complications can occur years after

an acute episode of HUS. The most common infectious agents are Shiga toxin producing enterohemorrhagic Escherichia coli (STEC) and sterp. pneumoniae.

\section{Shiga toxin-mediated hemolytic uremic syndrome:}

Shigella dysenteriae and some serotypes of Escherichia coli such as O157:H7 and O104:H4 produce Shiga toxin specifically Shiga toxin 1 (ST-1) and/or Shiga toxin 2 (ST-2), which are responsible for direct damage to renal epithelial, mesangial and vascular endothelial cells. STEC HUS is characterized by bloody diarrhea. However, diarrhea may also be present in some aHUS cases. Therefore, a diagnosis of STEC-HUS cannot be made on symptoms alone, and the earlier nomenclature that used "D+HUS" to correspond with STEC-HUS and

"D-HUS" to correspond with aHUS is not used at present. The involvement of Shiga toxins should be confirmed by, stool culture for shiga toxin producing E.coli(STEC), real time PCR for shiga toxin(Stx) genes, or the detection of anti-lipopolysaccharide-IgM antibodies against common STEC serogroups 8 . STEC can trigger HUS episode in approximately $1 \%$ of patients with complement mutation (mostly membrane co factor protein (MCP) mutation in children).

Early supportive treatment and dialysis are the main stay of treatment. The role of antibiotics in E.Coli infection is debated as this may induce expression of shiga toxin and increases risk of HUS. However, patients with shigellosis require prompt antimicrobial treatment in order to reduce mortality. Ciprofloxacin is recommended by WHO as first line therapy while ceftriaxone, azithromycin and cefixime are alternate agents. ${ }^{8.9}$

Figure 1: An etiology-based classification of the various forms of thrombotic microangiopathies. Reprinted by permission from Nature/Springer: Ref 2.

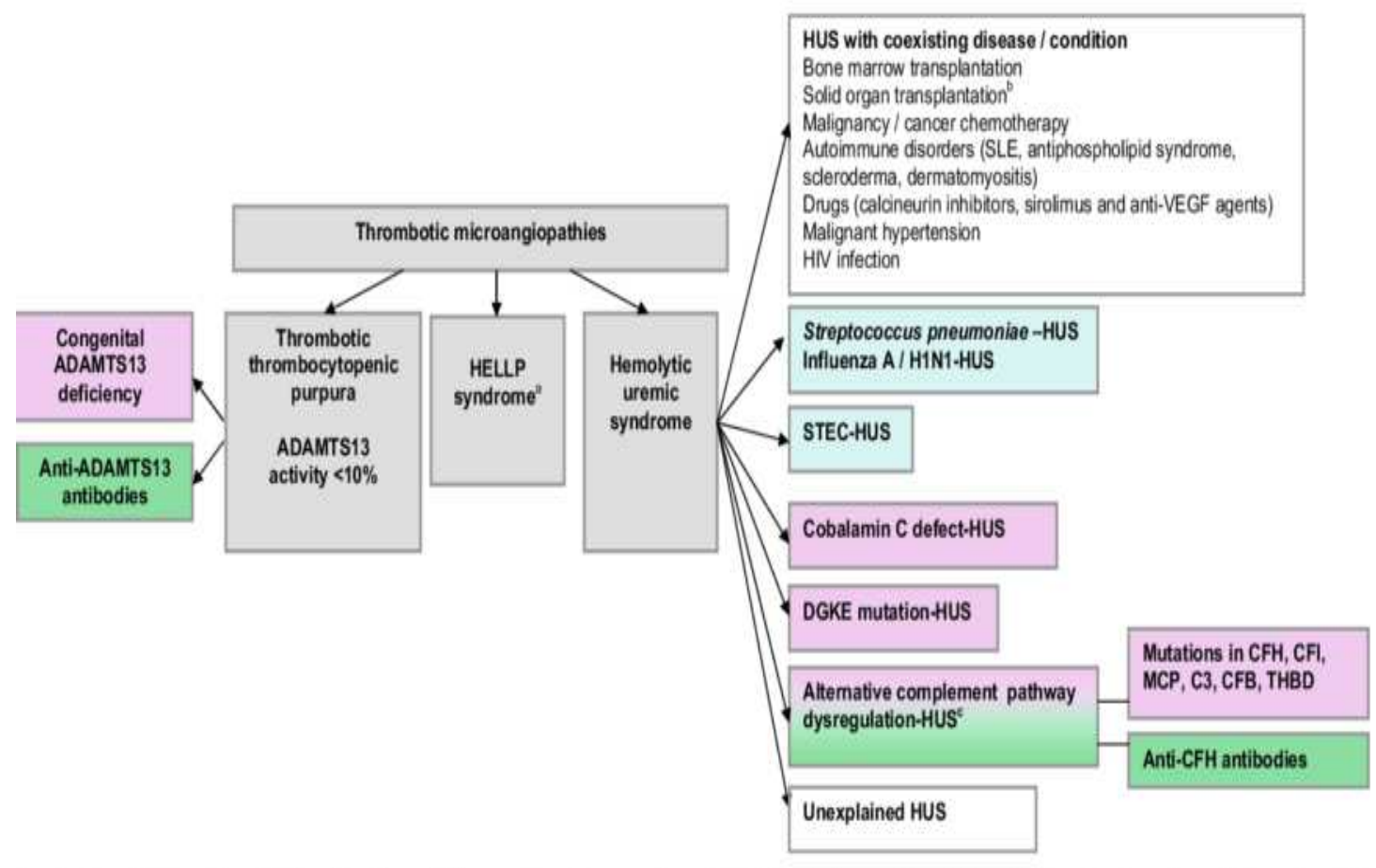

\section{Streptococcus pneumonia (S.P) induced HUS:}

HUS may occur following an invasive infection with Streptococcus pneumoniae. The preceding Streptococcus pneumoniae infection is usually severe and invasive, and children may present with septicemia, meningitis, and/or pneumonia with empyema. ${ }^{10}$ The mechanism of cellular injury in SP-associated HUS is thought to be related to exposure of the normally hidden Thomsen-Friedenreich (T) antigen, by circulating neuraminidase produced by the Streptococcus pneumonia organism. The neuraminidase removes the $\mathrm{N}$-acetylneuraminic acid, and normally circulating anti-T-antigen antibodies react with the newly exposed $\mathrm{T}$ antigen on red blood cells, platelets, and endothelial cells, precipitating hemolytic anemia, thrombocytopenia, and microvascular injury characteristic of HUS. Because blood products contain anti-T-antigen antibodies, plasma infusions should be minimized, and blood products should be washed so as to prevent administration of additional anti-T-antigen antibodies. 


\section{Cobalamin deficiency-associated HUS:}

Cobalamin deficiency-associated HUS is rare form of HUS, that occurs due to mutations in the MMACHC (methylmalonic aciduria and homocystinuria type $\mathrm{C}$ protein) gene, when a gene mutation is present, $\mathrm{MeCbl}$ will not metabolize homocysteine. Exactly how this metabolic change causes HUS is unclear. Suggested mechanisms include endothelial damage induced by hyperhomocysteinemia, impairment of the nitric oxide dependent inhibition of platelet aggregation, or induction of a procoagulant state of the endothelium leading to the formation of microthrombi.

This autosomal recessive disorder typically presents in the neonatal period with vomiting, poor sucking, failure to thrive, lethargy, hypotonia, thrombocytopenia, microangiopathic hemolytic anemia, and renal injury. ${ }^{12}$ Presentation may also occur in the older child. Adult onset is extremely uncommon though may occur. ${ }^{13}$ Regardless of age at presentation, neurologic symptoms are prominent and also include cognitive impairment, ataxia, and psychosis. Rapid deterioration of the patient leading to severe respiratory and hepatic failure has been reported. ${ }^{14}$ Renal disease is present in many. Megaloblastosis and leukopenia are unique features that are related to the metabolic defect responsive to low protein diet, Betaine, methionine and injectable vitamin B12.

Fig 2: Proposed diagram to differentiate atypical hemolytic uremic syndrome (aHUS) among thrombotic microangiopathies. Ref 5.

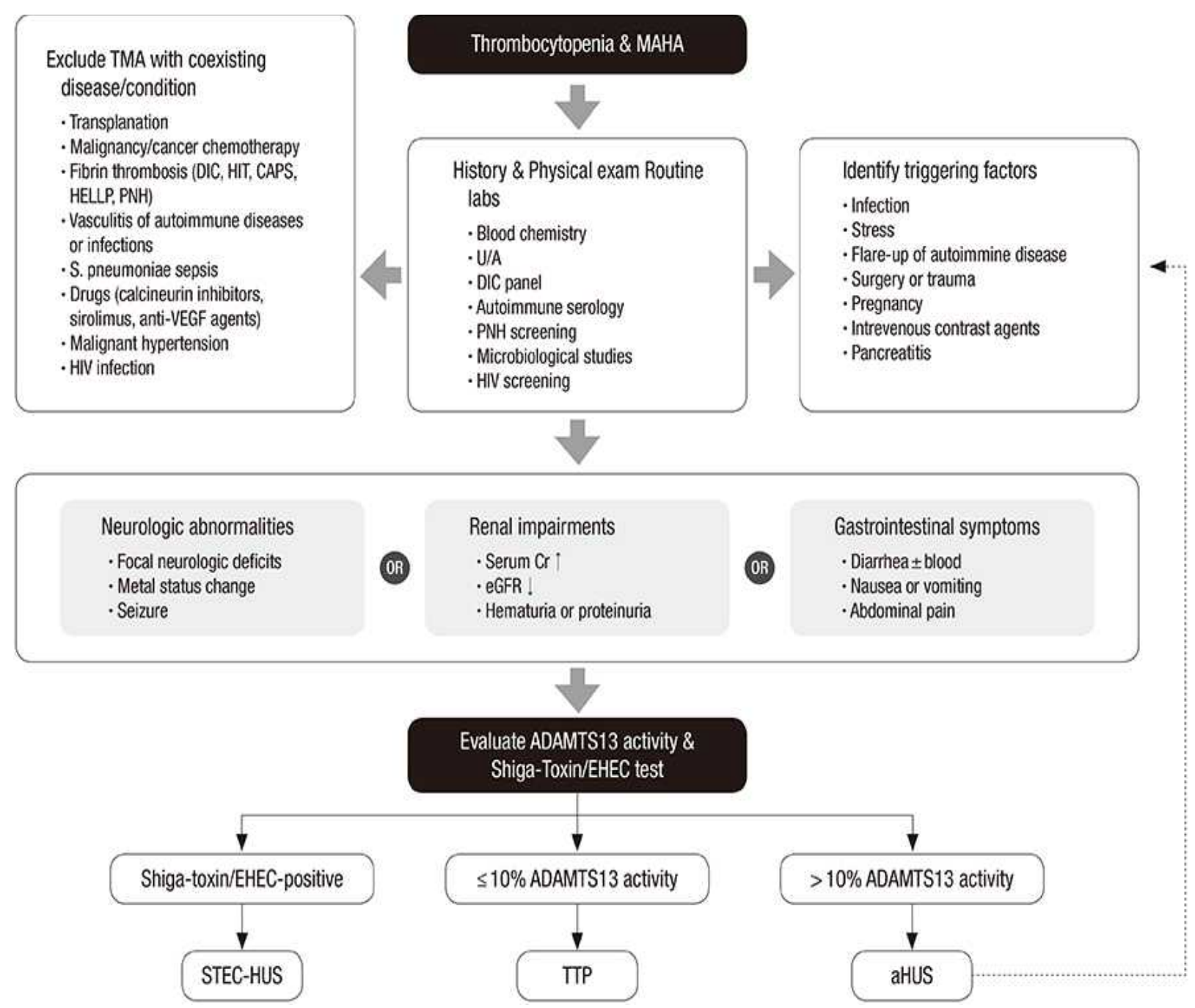




\section{Thrombotic Thrombocytopenia Purpura (TTP):}

Thrombotic thrombocytopenia purpura (TTP) is associated with abnormalities of von Willebrand factor-cleaving protease activity (ADAMTS13). It is rare in children. Patients with TTP have very low levels of ADAMTS 13 activity, typically less than $10 \%$ of normal.

${ }^{15}$ ADAMTS13 prevents the emergence and persistence of unusually large multimers of VWF by cleaving multimeric vWF from the endothelium and platelets into smaller multimers. Patients with TTP have unusually large multimeric forms of TTP in their plasma, which are able to agglutinate platelets under high sheer stress. ${ }^{16}$

The onset of symptoms in TTP can be gradual or sudden and can fluctuate and relapse. Neurologic involvement is usually central to the clinical manifestations and can wax and wane. Laboratory findings include the typical finding of hemolytic anemia, thrombocytopenia, and acute kidney injury; however, kidney injury is rarely severe. TTP is either inherited due to ADAMTS13 activity gene mutations or acquired due to anti-ADAMTS13 autoantibodies. ${ }^{17,18}$ When TTP is inherited symptoms may begin in the newborn period, while in TTP due to autoantibodies that interfere with ADAMTS 13 activity, the onset of symptoms can be in childhood or in adults. For the management start plasma infusion and plasmaphresis immediately. If ADAMTS13 is <5\% and anti-ADAMTS13 autoantibodies are negative continue only plasma infusion. However, if antibodies are positive continue with plasma exchange.

\section{Atypical HUS (aHUS):}

Atypical hemolytic uremic syndrome(aHUS) also called complement mediated or genetically mediated Hemolytic uremic Syndrome (HUS). The diagnosis of aHUS remains clinical. Microangiopathic hemolytic anemia and thrombocytopenia are the most common clinical manifestations of aHUS. It ultimately affects the kidneys and brain, leading to acute kidney injury and neurologic abnormalities such as diplopia, blindness, paresis, convulsion, confusion and coma. The disease may also invade the cardiovascular system, resulting in myocarditis, cardiomegaly, pericardial effusion, myocardial infarction, cardiac failure and even distal gangrene. The most common sequelae resulting from aHUS are chronic kidney disease and hypertension. ${ }^{19} \mathrm{~A}$ preceding illness, particularly a respiratory illness or a gastrointestinal infection (causing non-bloody diarrhea), is often reported Much less commonly, aHUS may be more indolent, with only marginally low platelet levels and a less substantial anemia than is classically seen in STEC HUS. ${ }^{20}$

Abnormalities of the complement regulation system is the main cause of aHUS and include various kinds of mutations and copy number variations in the genes encoding $\mathrm{C} 3, \mathrm{CFH}, \mathrm{CFI}, \mathrm{CD} 46 /$ membrance Co factor protein,(MCP), CFB, thrombomodulin (THBD), diacylglycerol kinase $\varepsilon$ ( DGKE) and complement factor $\mathrm{H}$ - related proteins 1 through 5 (CFHR1-5) or anti-CFH antibodies. ${ }^{21}$ Currently, $60 \%-70 \%$ of patients with aHUS have identifiable mutations in complement genes or anti-CFH antibodies. ${ }^{22}$

Fig 3: Alternate Complement Pathway Proteins. Ref 5

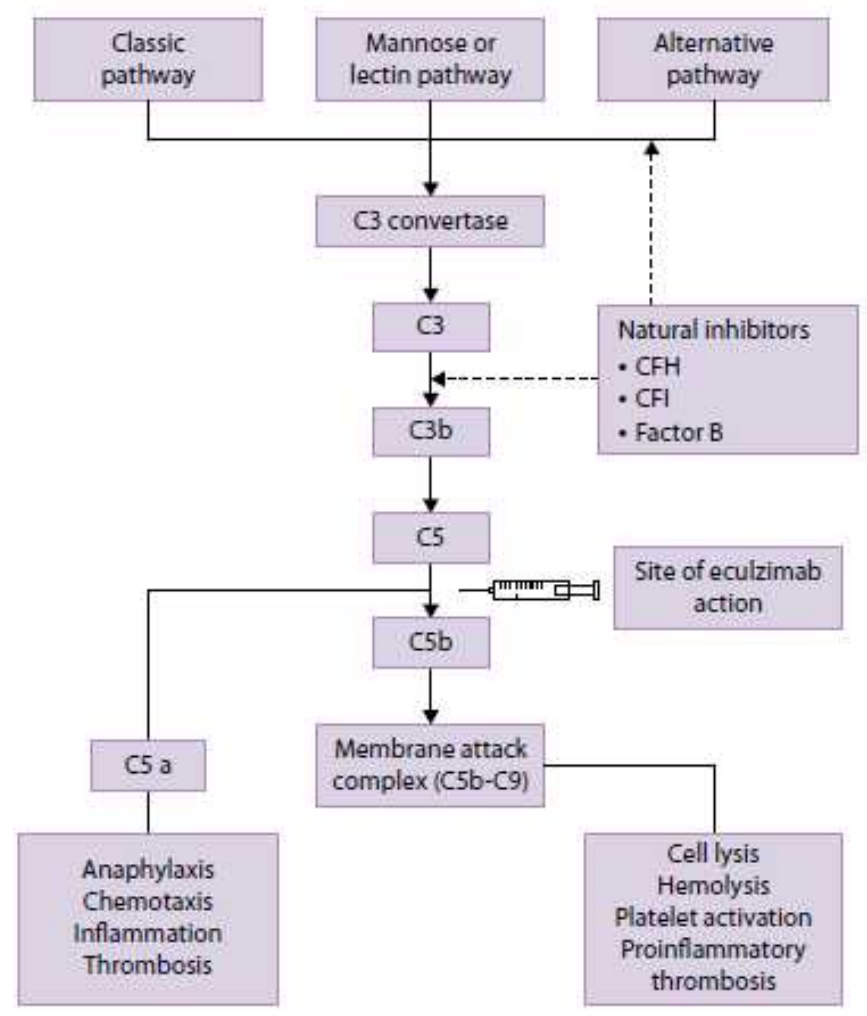




\section{HUS \& Pediatric Population}

The complement system consists of 30 plasma and cell surface - bound proteins. Complement activation is initiated by three pathways: Classical pathway is activated by immune complexes, Lectin pathway results from protein interactions with pathogens and Alternative pathway is a positive feedback loop that is constitutively active due to spontaneous hydrolysis of C3.This enables a rapid response against pathogens, but leaves the host vulnerable to damage if the amplification loop is unchecked. The system is therefore tightly regulated by plasma proteins: Factor $\mathrm{H}$ and factor I and cell surface proteins: membrane cofactor protein (CD46) (Fig 3). ${ }^{23}$

Defects in these regulators lead to complement dysregulation, activation of the terminal complement pathway and generation of the anaphylatoxin C5a and the membrane attack complex (C5b-9), resulting in a complement mediated aHUS. In each form of HUS, capillary and arteriolar injury in kidney leads to localized thrombosis, particularly in glomeruli, causing direct decrease in glomerular filtration. Progressive platelet aggregation in these areas of microvascular injury results in consumptive thrombocytopenia. Microangiopathic hemolytic anemia results from mechanical damage to red blood cells as they pass through damaged and thrombotic microvasculature, Fig $44^{24}$

\section{Diagnosis:}

Atypical HUS is suspected in patients with TMA having ADAMTS13 activity $>10 \%$, without evidence of STEC-HUS or secondary cause of HUS. In addition, it should also be strongly suspected if: very young age at onset ( $<6$ months); time point of HUS onset is not clear; anemia of unknown cause; recurrent HUS; recurrence of HUS after renal transplantation; family history of HUS and absence of diarrhea or bloody stools. ${ }^{25}$

Complement levels should be measured for all patients. If levels are decreased, then aHUS should be suspected however, a normal C3 level does not rule out diagnosis of aHUS. Likewise, decreased CFH or complement factor I (CFI) are observed in approximately $50 \%$ and $30 \%$ of patients with mutated CFH or CFI, respectively. Therefore, a normal CFH or CFI plasma level does not exclude a mutation in the corresponding gene. ${ }^{26}$

Genetic work up is recommended for all patients with aHUS who have no coexisting disease/condition, STEC infection, severe ADAMTS 13 deficiency, or hyperhomocysteinemia/methyl-malonic aciduria. In addition, they should be performed without delay if the patient has HUS relapse, a family history of non-synchronous HUS, or de novo post-transplant HUS and essentially before renal transplantation for aHUS. However, it is not justified before transplantation for STEC-HUS, unless this diagnosis is uncertain or unproven. ${ }^{19}$ Lastly, screening for DGKE mutation should also be performed in children with onset of aHUS before the age of $1-2$ years. ${ }^{27,28}$

Fig 4: The alternate Complement Pathway in atypical HUS and the associated genetic mutations. Ref 24.

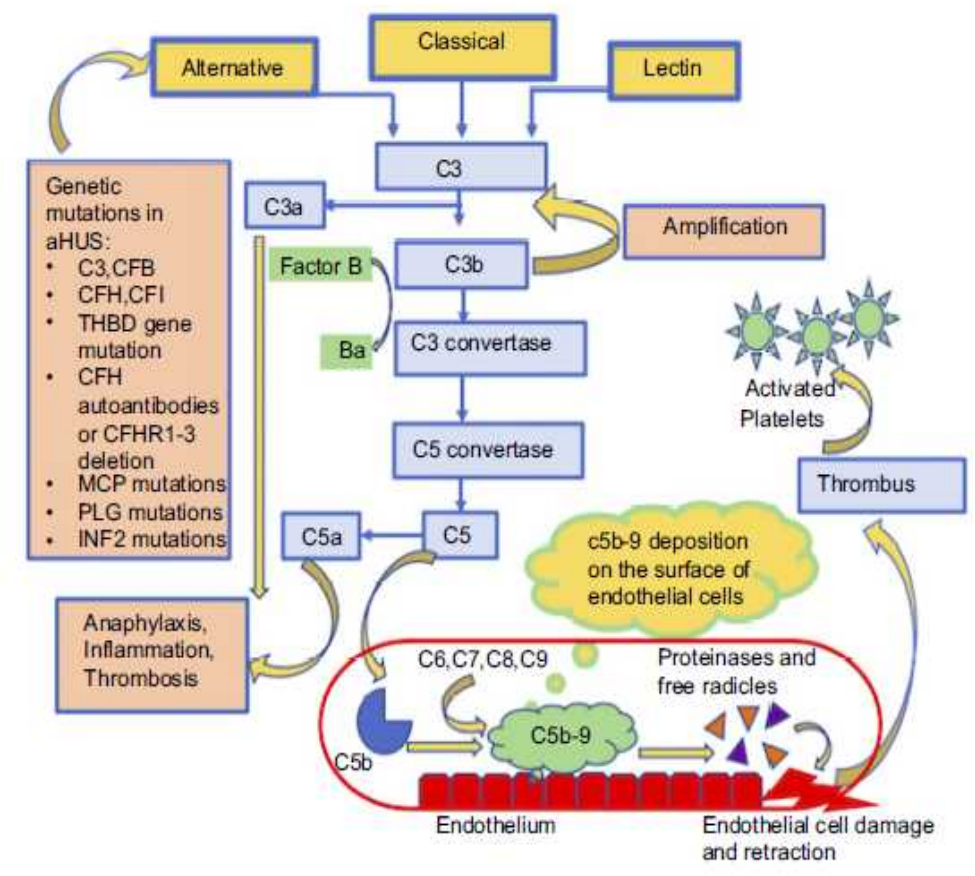

Figure 2 The alternative complement pathway in atypical hemolytic uremic syndrome and the associated genetic mutations.

Note: Daa from references 3, 16, 18-25.

Abbreviations: CFB, complement factor $\mathrm{B} ; \mathrm{CFD}$, complement factor $\mathrm{D} ; \mathrm{CF}$, complement factor $\mathrm{l} ; \mathrm{CFH}$, complement factor $\mathrm{H} ; \mathrm{MCP}$, membrane cofactor protein; THBD,

thrombomodulin; MAC, membrane attack complex; CFHR, complement factor receptor: PLG, plas minogen; INF2, Imverted formin 2; aHUS, atypical hemolytic uremic syndrome. 
Screening for mutations in $C F H, C F I, M C P, C 3, C F B$, THBD, and DGKE should be performed by direct Sanger sequencing analysis or next-generation sequencing (NGS), and screening for the $C F H$ hybrid gene and copy number variation in $C F H$ and $C F H R S$ should be performed by multiplex ligation-dependent probe amplification (MLPA) ${ }^{29}$ In addition, $6 \%-10 \%$ of aHUS patients have antibodies that bind to the $\mathrm{C}$ terminal region of factor $\mathrm{H}$, and as the prevalence of anti- $\mathrm{CFH}$ in Korean children is higher than in other countries, autoantibodies against factor $\mathrm{H}$ should be performed in an appropriately accredited laboratory. ${ }^{30-32}$ An international consensus has been published by Loirat et al for diagnostic purposes, Fig5. ${ }^{2}$

\section{Management:}

Supportive management is the basis of therapy in all forms of HUS. Infection can trigger relapse of aHUS so it should be treated appropriately. ${ }^{33}$ All patients who are clinically suspected of having aHUS should be offered a trial of Plasma exchange (PEx) and/or plasma infusion (PI) as early as possible. ${ }^{29}$ The theory behind the use of either of these interventions' hinges on the concept of either removing an abnormally functioning protein or replacing a deficient one. ${ }^{19} \mathrm{PEx}$ is an alternative option if eculizumab is not available, with an exchange of 1.5 plasma volumes (40-60 ml/kg) per session and with fresh-frozen plasma (FFP) for compensation. When PEx cannot be performed, PI (10-20 $\mathrm{ml} / \mathrm{kg})$ should be administered.

Fig 5 Diagnostic algorithm for atypical HUS in Children. Reprinted by permission from Nature/Springer: Ref 2.

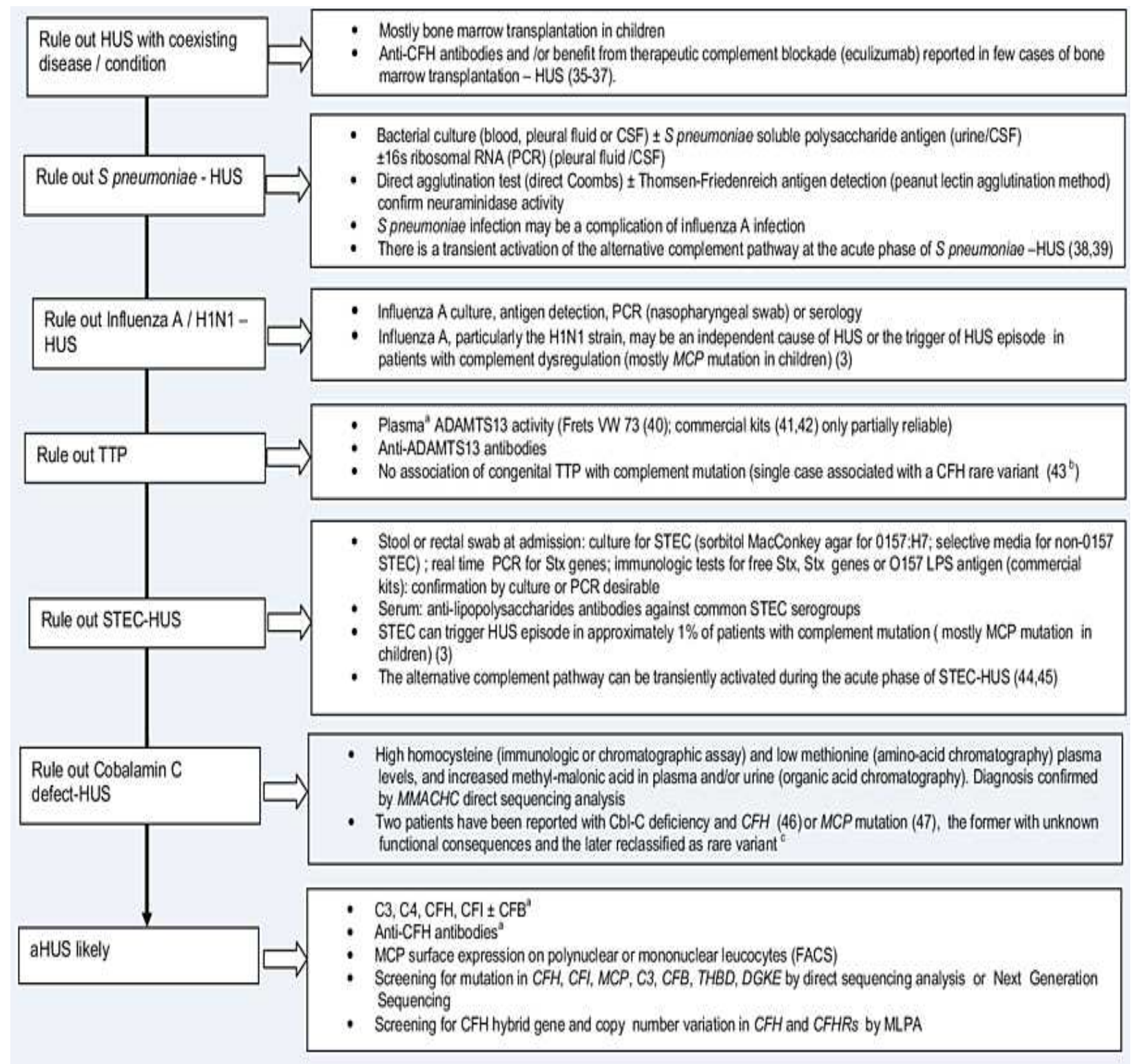

Patients receive PEx or PI daily from 5 days up to 2 weeks during the acute phase until the platelet count, lactate dehydrogenase (LDH), and hemoglobin levels have normalized and the renal function shows signs of improvement. When disease activity is controlled by daily 


\section{HUS \& Pediatric Population}

PEx, the subsequent frequency is 5 times per week for 2 weeks, and then 3 times per week for the subsequent 2 weeks. ${ }^{22}$ The frequency may be reduced from weekly to every 2 to 4 weeks as a long-term maintenance therapy. The further frequency of plasma therapy has to be decided according to the genetic information and clinical response to plasma therapy. In patients with an $M C P / C D 46$ mutation, the withdrawal of plasma therapy is possible because MCP is not a circulating protein. An attempt to withdraw plasma therapy can be considered in patients without a relapse of HUS, despite tapering of PEx/PI to monthly administration. ${ }^{34}$ Although PEx/PI induced remission in 55\%-80\% of episodes in patients with aHUS in the Italian cohort, $48 \%$ of children and $67 \%$ of adults died or progressed to ESRD during the 3-year follow-up period. ${ }^{19}$

Given what is known about the pathophysiology of aHUS, anticomplement therapy has become an option for the treatment of this disease. Eculizumab (Sirolis, Alexion Pharma), a monoclonal humanized anti-C5antibody, prevents C5 cleavage and formation of both C5a and C5b-9. ${ }^{35}$ Therefore, eculizumab therapy can reduce anaphylatoxin-induced inflammation and limit C5b-9 prothrombotic consequences of complement Activation ${ }^{36}$. The dosage and schedule of eculizumab should be adjusted according to body weight, as in Table $1 .{ }^{37}$ Whereas improvement of thrombocytopenia and elevated LDH occur more quickly after eculizumab therapy, recovery of renal function and other end-organ injuries present may take longer. Therefore, recovery from thrombocytopenia is used as a surrogate marker for activity of complement-mediated TMA. With continuous therapy, a time-dependent improvement of the estimated GFR is usually observed. Some patients eventually discontinued their previous dialysis. Patients with aHUS who undergo eculizumab therapy have an approximately $50 \%$ lower risk of reaching ESRD within 3 months of an aHUS episode compared with historical controls. ${ }^{38}$

Table 1: Weight-based dosing of Eculizumab in the treatment of aHUS. Ref 37

\begin{tabular}{lcc} 
Body weight & Induction period & Maintenance period \\
\hline$\geq 40 \mathrm{~kg}$ & $900 \mathrm{mg} / \mathrm{wk} \times 4$ doses & $\begin{array}{c}1,200 \mathrm{mg} \text { during week 5, } \\
\text { then 1,200 mg every 2 wk } \\
900 \mathrm{mg} \text { during week 3, } \\
\text { then } 900 \mathrm{mg} \text { every 2 wk }\end{array}$ \\
From $30 \mathrm{~kg}$ to $<40 \mathrm{~kg}$ & $600 \mathrm{mg} / \mathrm{wk} \times 2$ doses & $\begin{array}{c}600 \mathrm{mg} \text { during week 3, } \\
\text { then } 600 \mathrm{mg} \text { every 2 wk }\end{array}$ \\
From $20 \mathrm{~kg}$ to $<30 \mathrm{~kg}$ & $600 \mathrm{mg} / \mathrm{wk} \times 2$ doses & $\begin{array}{c}300 \mathrm{mg} \text { during week 2, } \\
\text { then } 300 \mathrm{mg} \text { every 2 wk }\end{array}$ \\
From 10 kg to $<20 \mathrm{~kg}$ & $600 \mathrm{mg} / \mathrm{wk} \times 1$ dose & $\begin{array}{c}300 \mathrm{mg} \text { during week 2, } \\
\text { then 300 mg every 3 wk }\end{array}$ \\
From $5 \mathrm{~kg}$ to $<10 \mathrm{~kg}$ & $300 \mathrm{mg} / \mathrm{wk} \times 1$ dose &
\end{tabular}

aHUS, atypical hemolytic uremic syndrome.

Patients should receive meningococcal vaccination at least 2 weeks prior to the first dose of eculizumab because the drug increases the risk of meningococcal infection. If eculizumab treatment is initiated less than 2 weeks after vaccination, patients should receive additional prophylactic antibiotics until 2 weeks after vaccination. ${ }^{39}$

It is important to emphasize that any complement-activating clinical event (infection, surgery, or pregnancy) may precipitate another acute episode of aHUS, careful monitoring and counseling should be part of the treatment plan for patients who have discontinued eculizumab. ${ }^{40}$ Ravulizumab, a long-acting C5 inhibitor, was approved by the Food and Drug Administration for the treatment of adult and pediatric patients $\geq 1$ month of age with aHUS to inhibit complement-mediated TMA. Ravulizumab treatment of aHUS should be maintained for a minimum duration of 6 months; extension beyond 6 months should be determined on an individual basis. Ravulizumab reduces the treatment burden, with infusions limited to once every 4 or 8 weeks depending on body weight. ${ }^{41}$

Special consideration may be given to the treatment of aHUS triggered by antibodies to FH. A number of authors have reported that the risk of having recurrent disease in this setting is based on the FH antibody titer (provided no mutations are present). Therefore, it has been proposed that an acceptable treatment plan for antibody-mediated aHUS includes an antibody reduction strategy. This entails either use of plasma exchange or anti-cellular immune suppression (cyclophosphamide, rituximab, or mycophenolate) in order to reduce the antibody titer to a safe level. ${ }^{42}$

\section{Liver transplantation:}

A number of the complement proteins are manufactured in the liver (FH, FI, C3, and FB). An isolated liver transplantation or a combined liver and kidney transplantation may be an option for patients with preserved eGFR, despite a severe and/or relapsing course, and for patients with a $C F H, C F I, C F B$, or $C 3$ mutation, complications, or no benefit from PEX/PI and no access to eculizumab treatment. ${ }^{43}$ 


\section{Kidney transplantation}

The risk of HUS recurrence after kidney transplantation varies according to the underlying genetic abnormality. Renal transplantation alone is not recommended for patients with a $C F H$ or $C F I$ mutation because of the poor outcome, with $80 \%$ of patients losing their graft due to a recurrence of the disease within 2 years. In patients with an $M C P / C D 46$ mutation alone, the risk of recurrence post-transplantation is low. Patients with a $C 3$ or $C F B$ mutation show a significant risk of disease recurrence post-transplantation. No post-transplantation recurrence has been observed to date in patients with a $D G K E$ mutation. Patients with an anti-factor $\mathrm{H}$ autoantibody should be treated with a PEX combination with Rituximab to minimize the antibody titer before proceeding to renal transplantation. ${ }^{29}$

Living-related renal transplantation alone should be avoided in cases of aHUS. PEX/PI for post-transplant recurrence usually failed to prevent graft loss, and prophylactic PEX/PI was recommended. According to the Consensus Study Group, one PEX with FFP (60-75 $\mathrm{ml} / \mathrm{kg}$ ) should be performed within 4-6 hours before graft reperfusion, FFP $(10-20 \mathrm{ml} / \mathrm{kg})$ should be infused during surgery, and PEX with FFP (60-75 ml/kg) should be continued daily for at least 5 days, followed by 5 sessions per week for 2 weeks, and then 3 sessions per week for 2 weeks, after which it should be tapered on a case-by-case basis. Recently, prophylactic eculizumab treatment for patients at high risk for post-transplant recurrence has been considered. ${ }^{44}$

\section{Conclusion and Suggestions:}

The diagnosis and management of HUS is challenging but is essential because of devastating consequences of inadequate treatment. Though better understanding of pathogenesis has a considerable impact on treatment modalities and outcome, the scenario is not so encouraging in developing countries. Followings are suggestions which can helpful in clinching diagnosis and subsequent management.

In resource limited countries, triad of microangiopathic non immune hemolytic anemia( $\mathrm{Hb}<10 \mathrm{gm} / \mathrm{dl})$ with high LDH, low haptoglobin, indirect hyperbilirubinemia and presence of schistocytes (up to 10\%) on peripheral smear ,thrombocytopenia (Plt $<150,000 / \mu \mathrm{L}$ and acute kidney injury( S/Creatinine 1.5 times higher than reference value for age and gender) is sufficient to label the patient as HUS.

The distinction between various causes is important because the clinical course, treatment and prognosis differ for each category. Presence of bloody dysentery will give clue towards STEC - HUS. As detection of toxins and serological tests is not available, stool culture for enterohaemorrhagic E.Coli, shigella and salmonella may be helpful if samples are collected before initiating treatment.

A high index of suspicion must be kept for aHUS in asynchronous, non diarrhea associated HUS, as in a local study 25 cases have reported over a period of 15 months. ${ }^{45}$ (sample sent to Boston Medical School for genetic work up). Exclusion of TTP by measuring ADMTs 13 is not mandatory as TTP is rare in children. However, DIC, malaria and autoimmune disorders need to be ruled out.

Clinical findings like septicemia, meningitis, and/or pneumonia with empyema will be suggestive of strep. Pneumoniae infection, whereas neurologic symptoms, megaloblastosis and leukopenia are unique features that are related to the metabolic defect of cobalamin. Supportive management is the basis of therapy in all forms of HUS. Meticulous care of fluid and electrolyte disturbances especially fluid overload once AKI sets in. Early dialysis should be instituted when required. Platelet transfusion should be avoided unless platelet count is $<10,000 /$ ul or to enable vascular catheter insertion. Blood transfusions is recommended for patients with hemoglobin $6<\mathrm{gm} / \mathrm{dl}$ or hemodynamic instability.

Since, genetic analysis for mutation is not available locally, all patients clinically suspected of having aHUS should be treated in specialized centres with facility for dialysis and/or plasma exchange. They should be offered a trial of PEX and/or plasma infusion (PI) as early as possible. PEX is an alternative option if eculizumab is not available.

The facility for transplant is available in couple of centers, isolated liver transplantation or a combined liver and kidney transplantation may be offered wherever indicated. Last but not the least, resources need to be diverted to improve diagnostic facilities and to provide optimal infra structure to make a difference in terms of morbidity and mortality.

\section{Conflict of Interest: None declared}

\section{References:}

1. Ellis AD, William H E, Niaudet PD, Norishige Y, Francesco E , Stuart G L.. Hemolytic Uremic Syndrome. In: Pediatric Nephrology. $7^{\text {th }}$ ed. Verlag Berlin Heidelberg :Springer;2016:1409-1513

2. Loirat C, Fakhouri F, Ariceta G, Besbas N, Bitzan M, Bjerre A et al. An international consensus approach to the management of atypical hemolytic uremic syndrome in children. Ped Nephrol.2016;31:1539

3. Noris M, Remuzzi G. Atypical hemolytic-uremic syndrome. New Engl J Med. 2009; 361 (17): 1676 - 87.

4. Lee H, Kang E, Kang H G, Kim Y H, Kim JS, Kim Hee-J et al. Consensus regarding diagnosis and management of atypical hemolytic uremic syndrome. Korean J Intern Med. 2020;35:25-40

5. Cheong H II, Jo S Kyung, Yoon S-S, Cho H, Kim J S, Kim Y Ok et al. Clinical Practice Guidelines for the Management of Atypical Hemolytic Uremic Syndrome in Korea . J Kor Med Sci. 2016; 31: 1516-1528

6. De Jorge EG, Pickering MC. Atypical hemolytic uremic syndrome: telling the difference between H and Y. Kidney Int 2010;78(8):721-3.

7. Bagga A, Khandelwal P, Mishra K, Thergaonkar R, Vasudevan A, Sharma J et al. Hemolytic uremic syndrome in developing countries: Consesus guidelines. On behalf of the Indian Society of Pediatric Nephrology Hemolytic uremic syndrome in a developing country: Pediatric Nephrology https://doi.org/10.1007/s00467-019-04233

8. Williams PCM, Berkley JA . Guidelines for the treatment of dysentery (shigellosis): a systematic review of the evidence. Paed Int Child Health. 2018; 38:S50 - S65 


\section{HUS \& Pediatric Population}

9. WHO (2017) The selection and use of essential medicines. www. who.int/medicines/publications/essentialmedicines/en/

10. Copelovitch L, Kaplan BS. Streptococcus pneumoniae-associated haemolytic uremic syndrome. Ped Nephrol. 2008;23:1951-1956.

11. Cochran JB, Panzarino VM, Maes LY, Tecklenburg FW. Pneumococcus-induced T-antigen activation in hemolytic uremic syndrome. Ped Nephrol. 2004:19:317 - 321.

12. Kind T, et al. Cobalamin C disease presenting as hemolytic-uremic syndrome in the neonatal period. J Ped Hem Onc. 2002;24(4):327-329.

13. Geraghty MT, et al. Cobalamin C defect associated with hemolytic-uremic syndrome. J Ped. 1992; 120(6):934 - 7. 219.

14. Carrillo-Carrasco N, Chandler RJ, Venditti CP. Combined methylmalonicacidemia and homocystinuria, cblC type. I. Clinical presentations, diagnosis and management. J Inherit Metab Dis. 2012;35(1): 91 - 102.

15. Loirat C, Girma JP, Desconclois C, Coppo P, Veyradier A. Thrombocytopenic purpura related to severe ADAMTS13 deficiency in children. Ped Nephrol. 2009;24:19 - 29 .

16. Moake JL, Byrnes JJ, Troll JH, Weinstein MJ, Colannini MN, Azocar J, et al. Unusually large plasma factor VIII: von Willebrand factor multimers in chronic relapsing thrombotic thrombocytopenia purpura. N Engl J Med. 1982;307:1432 - 5.

17. Tsai HM, Chun-Yet LE. Autoantibodies to von Willebrand factor-cleaving protease in acute thrombotic thrombocytopenia purpura. N Engl J Med. 1998;338:1585 - 94

18. Levy GC, Nichols WC, Lian EC, Foroud T, et al. Mutations in a member of the ADAMTS13 gene family cause thrombotic thrombocytopenia purpura. Nature. 2001;413:488 - 94.

19. Sallee M, et al. Myocardial infarction is a complication of factor H-associated atypical HUS. Nephrol Dial Transplant. 2010;25(6):2028 - 32.

20. Noris M, et al. Relative role of genetic complement abnormalities in sporadic and familial aHUS and their impact on clinical phenotype. Clin J Am Soc Nephrol.2010;5(10):1844 - 59.

21. Sellier-Leclerc AL, et al. Differential impact of complement mutations on clinical characteristics in atypical hemolytic uremic syndrome. J Am Soc Nephrol. 2007;18(8):2392 - 400.

22. Stahl AL, Vaziri-Sani F, Heinen S, Kristoffersson AC, Gydell KH, Raafat R et al. Factor H dysfunction in patients with atypical hemolytic uremic syndrome contributes to complement deposition on platelets and their activation. Blood .2008; 111: 5307-5315.

23. Kher K, Schnaper HW, Makker SP. Clinical pediatric nephrology.London,England: CRC Press Ltd; 2006.

24. Raina R, Grewa M K 1, Radhakrishnan Y, TatineniV, DeCoy M. Burke L LG et al. Optimal management of atypical hemolytic uremic disease: challenges and solutions. Int J Nephrol Renovasc Disease.2019;12: 183 - 204

25. Sawai T, Nangaku M, Ashida A, Fujimaru R, Hataya H, Hidaka Y, et al. Diagnostic criteria for atypical hemolytic uremic syndrome proposed by the Joint Committee of the Japanese Society of Nephrology and the Japan Pediatric Society. Ped Int.2014; 56: 1-5.

26. Bento D, et al. Triggering of atypical hemolytic uremic syndrome by influenza A ( H1N1). Ren Fail. 201032(6): 7536.

27. Berner R, et al. Hemolytic uremic syndrome due to an altered factor H triggered by neonatal pertussis. Ped Nephrol. 2002;17(3):190 - 2 .

28. Ermini L, et al. Common genetic variants in complement genes other than CFH, CD46 and the CFHRs are not associated with aHUS. Mol Immun.2012;49(4):640 - 8.

29. Dragon-Durey MA, et al. Clinical features of antifactor H autoantibody-associated hemolytic HUS. J Am Soc Nephrol. 2010;21(12):2180 2187.

30. Taylor CM, Machin S, Wigmore SJ, Goodship TH; working party from the Renal Association, the British Committee for Standards in Haematology and the British Transplantation Society. Clinical practice guidelines for the management of atypical haemolytic uraemic syndrome in the United Kingdom.Br J Haematol.2010; 148: 37-47.

31. Dragon-Durey MA, Loirat C, Cloarec S, Macher MA, Blouin J, Nivet H,etal. Anti-Factor H autoantibodies associated with atypical hemolytic uremic syndrome. J Am SocNephrol.2005; 16: 555-563.

32. Lee JM, Park YS, Lee JH, Park SJ, Shin JI, Park YH, Yoo KH, Cho MH, Kim SY, Kim SH, et al. Atypical HUS: Korean pediatric series. Ped Int.2015; 57: 431-438.

33. Dragon-Durey MA, Blanc C, Roumenina LT, Poulain N, Ngo S, Bordereau P, Frémeaux-Bacchi V. Anti-factor H autoantibodies assay. Methods Mol Biol.2014; 1100: 249-256.

34. Banjerjee S, Sharma J. Summary of hemolytic uremic syndrome in a developing country. Asian J Ped Nephrol. 2019; 2:71-74.

35. Fernandez GC, Te Loo MW, van der VeldenTJA, et al. Decrease of thrombomodulin contributes to the procoagulant state of the endothelium in hemolytic uremic syndrome. Ped Nephrol. 2003;18:1066 - 1068

36. Legendre CM, Licht C, Muus P, Greenbaum LA, Babu S, Bedrosian C, et al. Terminal complement inhibitor eculizumab in atypical hemolyticuremic syndrome. N Engl J Med.2013; 368: 2169-2181.

37. Alexion Pharmaceuticals Inc. Soliris (eculizumab):US prescribing information.4/2014. http://www.accessdata.fda.gov/drugsatfda. docs/label/2014/125166s368s380lbl.pdf

38. Cofiell R, Kukreja A, Bedard K, Yan Y, Mickle AP, Ogawa M, et al. Eculizumab reduces complement activation, inflammation, endothelial damage, thrombosis, and renal injury markers in aHUS. Blood 2015; 125: 3253-62.

39. Fakhouri F, Delmas Y, Provot F, Barbet C, Karras A, Makdassi R, , et al. Insights from the use in clinical practice of eculizumab in adult patients with atypical hemolytic uremic syndrome affecting the native kidneys: an analysis of 19 cases. Am J Kidney Dis.2014; 63: 40-48.

40. Cataland SR, Wu HM. How I treat: the clinical differentiation and initial treatment of adult patients with atypical hemolytic uremic syndrome. Blood.2014; 123: 2478-84.

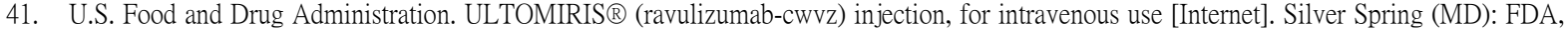
2019 [cited 2019 Dec 12]. Available from: https://www.accessdata.fda.gov/drugsatfda_docs/label/2019/761108s001lbl.pdf.

42. Hofer J, Giner T, Jozsi M. Complement factor H-antibody-associated hemolytic uremic syndrome: pathogenesis, clinical presentation, and treatment. SeminThrombHemost.2014; 40: 431-443.

43. Saland JM, Ruggenenti P, Remuzzi G; Consensus Study Group. Liver-kidney transplantation to cure atypical hemolytic uremic syndrome. J Am Soc Nephrol.2009; 20: 940-949.

44. Loirat C, Fremeaux- Bacchi V. Atpical hemolytic uremic syndrome. Orphanet J Rare Ds. 2011;6:60.

45. Ahmad J, Hafeez F, Shafi F, Akhtar N. Clinical spectrum, complications and outcome of atypical hemolytic uremic syndrome. Pak Armed Forces Med J.2020; 70(Supp1): S176-81. 